\title{
TRACE (Trunk Aesthetic Clinical Evaluation), a routine clinical tool to evaluate aesthetics in scoliosis patients: development from the Aesthetic Index (AI) and repeatability
}

\author{
Fabio Zaina*, Stefano Negrini and Salvatore Atanasio
}

Address: ISICO (Italian Scientific Spine Institute), Via Roberto Bellarmino 13/1, 20141 Milan, Italy

Email: Fabio Zaina* - fabio.zaina@isico.it; Stefano Negrini - stefano.negrini@isico.it; Salvatore Atanasio - salvatore.atanasio@isico.it

* Corresponding author

Published: 20 January 2009

Scoliosis 2009, 4:3 doi:10.1186/1748-7|6|-4-3

This article is available from: http://www.scoliosisjournal.com/content/4/I/3

(C) 2009 Zaina et al; licensee BioMed Central Ltd.

This is an Open Access article distributed under the terms of the Creative Commons Attribution License (http://creativecommons.org/licenses/by/2.0), which permits unrestricted use, distribution, and reproduction in any medium, provided the original work is properly cited.

\begin{abstract}
Background: Aesthetic appearance is of primary importance in the treatment of adolescent idiopathic scoliosis (AIS), but to date tools for routine clinical practice have not become available. The aim of the present study is to develop such a tool and to verify its repeatability.

Methods: Instrumentation: At first we developed the Aesthetic Index (AI), based on a three-point scale for asymmetry of the shoulders, scapulae and waist that we tested for 5 years. From this experience we developed another tool we called TRACE, the acronym of Trunk Aesthetic Clinical Evaluation; TRACE is a I2-point scale based on four sub-scales, shoulders (0-3), scapulae (0-2), hemi-thorax (0-2) and waist (0-4).

Population: Posterior-anterior (PA) photographs of one hundred-sixty AIS patients

Procedures: Each photograph was scored in two independent tests by four observers using Al, and subsequently TRACE.

Data analysis: Kappa statistical analysis and $95 \%$ level of agreement were used; we also identified the minimum significant change ( $95 \%$ confidence level).

Results: We found the intra- and inter-raters repeatability of Al to be fair. Three points out of seven was the minimum significant change between two different evaluations. For TRACE, intrarater repeatability was fair and inter-raters poor; but the minimum significant change was three (intra-rater), or four (inter-raters) out of twelve points.

Conclusion: Widening the scale from 7 (AI) to 12 points (TRACE) increased the clinical sensitivity to changes of the aesthetic scale, even if TRACE has only a fair repeatability. TRACE is a no-cost tool for routine clinical practice in AIS patients. Due to the absence of other comparable validated tools, once the inherent measurement error is known and understood, its routine clinical use by physicians is advised.
\end{abstract}




\section{Background}

Aesthetic appearance is a primary consideration in the treatment of scoliosis. This has been clearly stated in a consensus by SOSORT experts, in which aesthetic improvement has become the main goal of scoliosis treatment.[1] Orthopaedic surgeons share this view of the relevance of aesthetics; in a recent study concerning the importance of physical deformity of patients with adolescent idiopathic scoliosis, "the severity of deformity" consistently ranked as the most important clinical consideration when proposing surgical treatment to patients.[2] Some attempts to measure and monitor aesthetics have been made. Some questionnaires, such as SRS $22,[3]$ include domains concerning aesthetics, while some questionnaires have been designed and validated specifically to measure the perception of spinal deformity by the patient (or the parent). This is the case of the Walter Reed Visual Assessment Scale [4] and the more recently developed "Spinal Appearance Questionnaire" [5]. These instruments have the advantages of considering the patient's subjective judgement of his own aesthetics, but this does not correspond to the objective situation as can be judged by an external observer. This means that these are more psychological than aesthetic evaluation tools.

Various high-tech instruments for trunk surface evaluation are available, such as ISIS, Formetric, Quantec, AUSCAN and others [6-12], but none has reached any kind of consensus or is used extensively in routine clinical practice. This is particularly attributable to the high cost of such instruments, which limits availability to hospitals and clinics while hindering the integration of these instruments into standardized procedures. Attempts have been made to establish the inter-rater reliability of aesthetics clinical evaluation in AIS patients, but the results have not been satisfactory [13]. Thus, despite the judgment among physicians that aesthetics is of great importance in AIS patients, there are no clinical practice tools by which to assess its changes during treatment.

The objective of the present study was to develop an routine clinical tool and verify its intra- and inter-rater repeatability in the assessment of aesthetics in AIS patients.

\section{Methods}

For more than twenty years our group has evaluated the aesthetics of the posterior trunk, ranking the asymmetry of the shoulders, scapulae and waist (0 absent, 1 slight, 2 important) even if without giving to this evaluation a great importance. Facing the needs reported in the introduction, in the last five years we set out to build on our experience to develop a new clinical tool called the Aesthetic Index (AI), which corresponds to the sum of these three subscale scores.

\section{Population}

Posterior-anterior (PA) photographs of one hundred-sixty AIS patients

\section{Procedures}

Each photograph has been scored twice independently by four observers. In this way we had four pairs of intra-rater and four pairs of inter-rater evaluations. Each single observer performed the evaluation twice, and there was an interval of one week between observations.

\section{Data analysis}

We used Kappa statistics (0-0.2 poor, 0.2-0.4 fair, 0.40.6 moderate, $0.6-0.8$ good, $0.8-1.0$ very good), an index of the observer disagreement which compares the agreement found against that which might be expected by chance. Kappa can be thought of as the chance-corrected proportional agreement, and possible values range from +1 (perfect agreement) via 0 (no agreement above that expected by chance) to -1 (complete disagreement). Despite published controversies [14-16], Kappa statistics are still widely used. In particular, we are aware that Kappa may be low even though there are high levels of agreement and even though individual ratings are accurate. Whether a given kappa value implies a good or a bad rating system or diagnostic method depends on what model one assumes about the decision making of raters [17]. Accordingly we also used the Percent of agreement, the percentage of the answers that were equal in the two repeated measures. Moreover, for clinical purposes, we present the $95 \%$ level of agreement, or the number of points of difference needed to reach an agreement of $95 \%$. To give an example: for the rater who obtained the worst results the Percent of agreement for TRACE was 28.8\% (Table 1), but if the repeated measurements one point above (or below) are considered, a 99.4\% of agreement can be reached. This corresponds to a $95 \%$ of agreement of 2 points out of 12 . This result has an high clinical significance, because it means that, in everyday practice, considering two evaluations made by the same rater, a real change occurs only if the variation is over the $95 \%$ level of agreement, that in the example given corresponds to 3 points out of 12 .

\section{Second Study: TRACE}

Given the results from the first part of our study, we developed a new scale called TRACE, that is the acronym of Trunk Aesthetic Clinical Evaluation), for the purpose of improving the AI widening (and deepening) the scale.

\section{Instrumentation}

TRACE is based on four sub-scales: shoulders, scapulae and waist (which were already present in the AI), and the hemi-thorax (Fig 1, 2, 3, 4). However, the scores for each sub-scale were changed with respect to AI: shoulders now ranged from 0-3, waist from 0-4, scapulae from 0-2 and 
Table I: Results of statistical analysis of TRACE and its individual items

\begin{tabular}{|c|c|c|c|c|c|c|c|c|}
\hline & \multicolumn{2}{|c|}{ Kappa statistics range } & \multicolumn{2}{|c|}{ Percent of agreement } & \multicolumn{2}{|c|}{$95 \%$ level of agreement (range) } & \multicolumn{2}{|c|}{ Minimum Significant Change } \\
\hline & Intra-raters & Inter-raters & Intra-raters & Inter-raters & Intra-raters & Inter-raters & Intra-raters & Intra-raters \\
\hline TRACE & $0.16-0.24$ & $0.09-0.14$ & $28.8-36.3$ & $18.8-36 . \mid$ & $2 / 12(99.4-96.9 \%)$ & $3 / 12(95.0-100 \%)$ & $3 / 12$ & $4 / 12$ \\
\hline Shoulders & $0.29-0.43$ & $0.16-0.25$ & $51.3-70.6$ & $48.8-70.6$ & $1 / 4(96.9-100 \%)$ & $1 / 4(92.5-100 \%)$ & $2 / 4$ & $2 / 4$ \\
\hline Scapulae & $0.43-0.58$ & $0.4 I-0.50$ & $76.9-79.4$ & $70.6-80.0$ & $1 / 3(99.4-100 \%)$ & $1 / 3(100-100 \%)$ & $2 / 3$ & $2 / 3$ \\
\hline Hemi-thorax & $0.22-0.41$ & $0.12-0.20$ & $58.8-63.1$ & $50.6-63.1$ & I/3 (98.I-99.4\%) & I/3 (95.6-99.4\%) & $2 / 3$ & $2 / 3$ \\
\hline Waist & $0.40-0.48$ & $0.07-0.11$ & $55.0-68.0$ & $24.4-68.1$ & I/5 (95.6-99.4\%) & $2 / 5(98.7-100 \%)$ & $2 / 5$ & $3 / 5$ \\
\hline
\end{tabular}

hemi-thorax from 0-2. From these sub-scales we calculated TRACE, using the sum of the sub-scale scores to reach a 12-point scale. These changes were based on our experience in using the AI. We realized that for the shoulders it was easy to define more intermediate values, so we defined asymmetry as slight (1), moderate (2) or important (3). For the waist it was easy to define a total asymmetry (a score of 4) when one flank was straight or when there was a lateral decompensation of the trunk. It was easy as well to define a very slight (a score of 1) and an important but not complete (a score of 3) asymmetry. Between these points we defined a mild asymmetry (a score of 2). The hemi-thorax item was created as a comple-
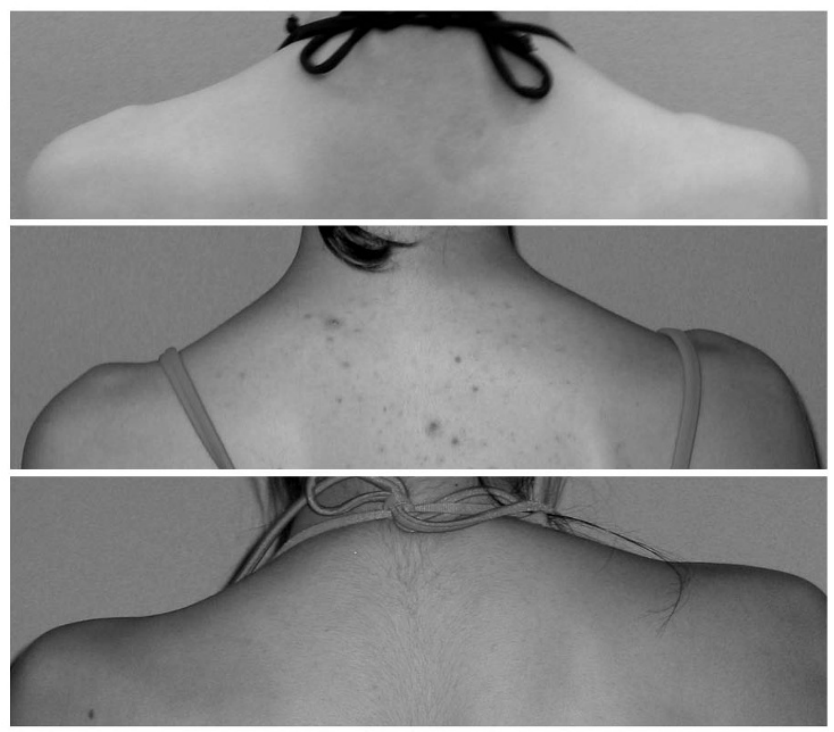

\section{Figure I}

Shoulder asymmetry, as evaluated in TRACE, ranges from 0 to 3. For the shoulders it is easy to detect some intermediate values, so we defined asymmetry (from the top) slight (I), moderate (2) and important (3). ment of the scapulae, since we noted that occasionally there is an evident prominence of the last ribs of the back even when there is no real asymmetry in the scapulae.

\section{Procedures}

The testing procedure of TRACE was similar to that of AI: The same 160 PA photographs of the trunks of AIS patients were evaluated by the same observers through the use of the same procedure.

\section{Data analysis}

The same statistical analysis used for AI was performed. We also correlated the AI scores with TRACE by comparing the values of the shoulders and waist, as well as the overall value of TRACE. The scapulae were not included in the analysis because the scores did not change.

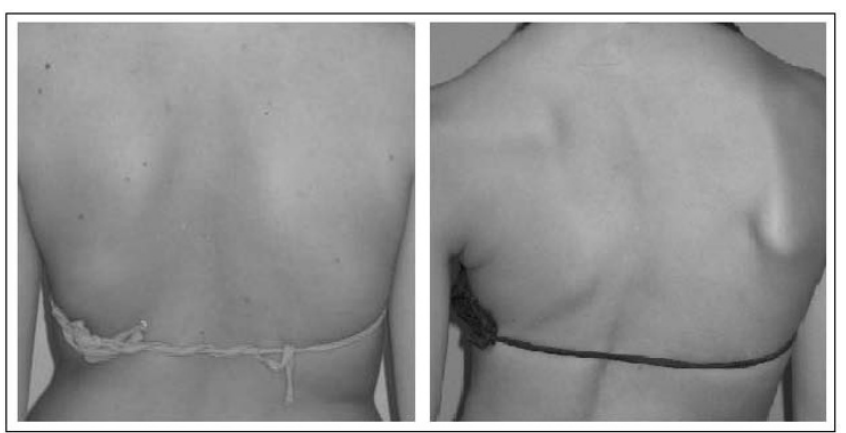

Figure 2

Hemi-thorax asymmetry as evaluated in TRACE: This item was created as a complement of the scapulae, since we noted that occasionally there is an evident prominence of the last ribs on the back even when there is no real asymmetry in the scapulae. From the left: slight (I) and important (2) asymmetry. 

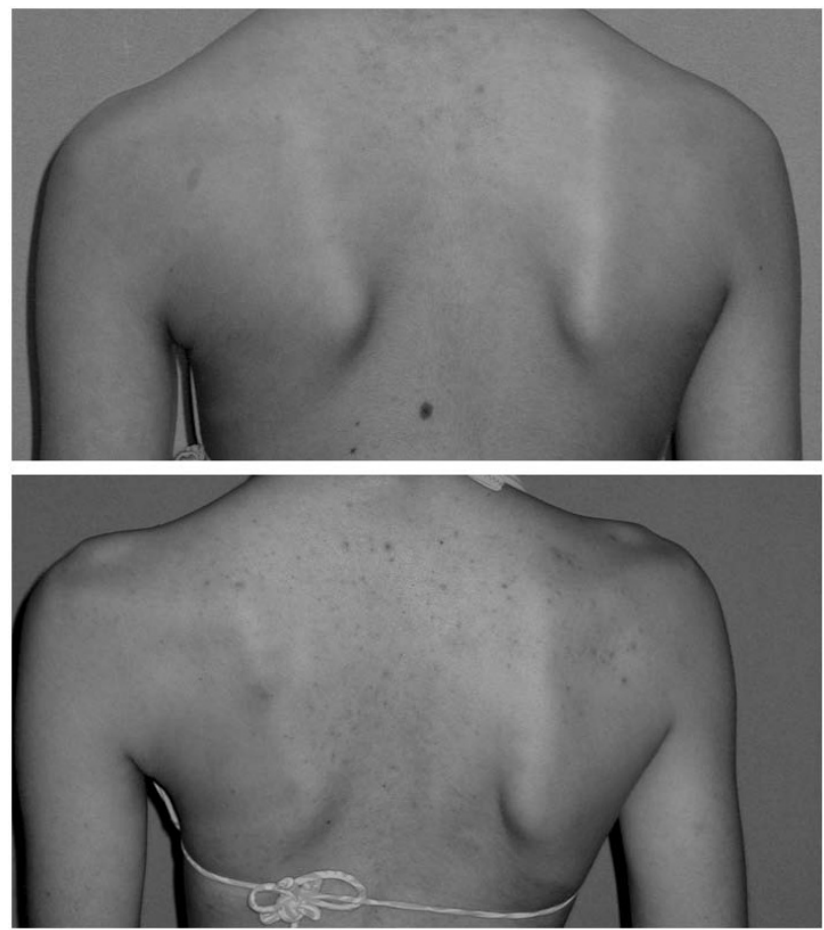

Figure 3

Scapulae asymmetry as evaluated in TRACE: (from the left) slight (I) and important (2).

\section{Results}

Regarding the AI, we found the repeatability of both intraand inter-raters to be fair (range of Kappa value 0.28-0.41 and $0.17-0.28$ respectively). The waist was the more reproducible sub-score, rating good and fair, respectively, for intra- and inter-raters (Table 2). At the 95\% level of agreement, we found that three points out of seven was the minimum change to be considered significant between two different evaluations both for the same and different raters.

Widening the scale with TRACE, we found intra-rater repeatability to be fair, while inter-raters were poor (Kappa value: $0.16-0.24$ and $0.09-0.14$ respectively; Table 1). All sub-scores graded as moderate for intra-rater, while the inter-rater was lower (moderate for the scapulae, poor for the waist and fair for the other subscores). At the 95\% level of agreement, we found that three points out of twelve was the minimum change to be considered significant between two different evaluations for the same rater, while four out of twelve was the minimum for different raters.

The correlations between the AI and TRACE are shown in the tables 3, 4 and 5 .
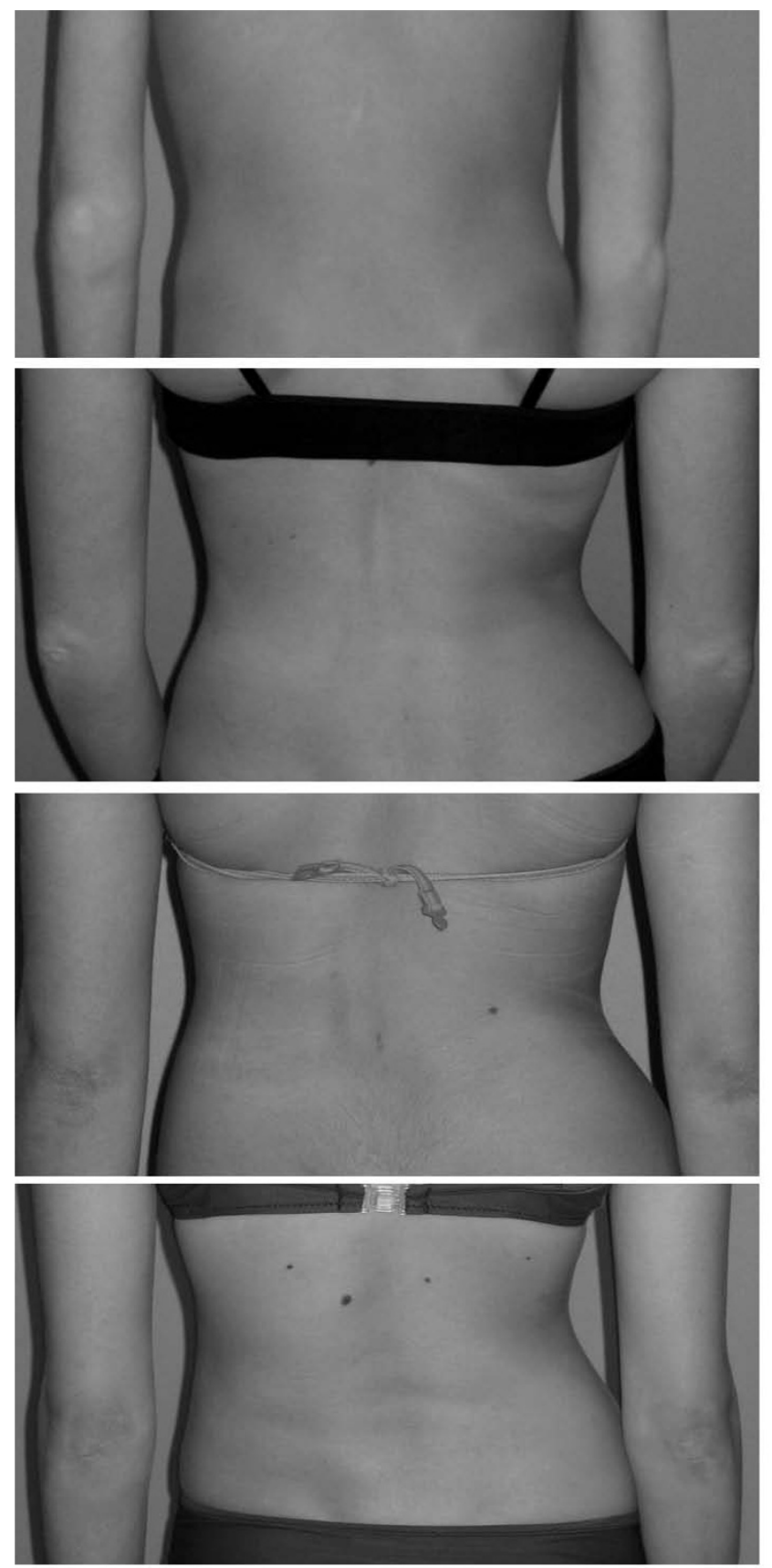

Figure 4

Waist asymmetry as evaluated in TRACE: it was quite easy to define a total asymmetry (a score of 4 ) when one flank was straight or when there was a lateral decompensation of the trunk. It was easy as well to define a very slight (a score of I) and an important but not complete (a score of 3 ) asymmetry; between these points we defined a mild asymmetry (a score of 2). In the figure, from the top: slight (I), mild (2), moderate (3) and important (4) asymmetry. 
Table 2: Results of statistical analysis of the Aesthetic Index and its individual items.

\begin{tabular}{lcccccccc}
\hline & \multicolumn{2}{c}{ Kappa statistics range } & \multicolumn{2}{c}{ Percent of agreement } & \multicolumn{2}{c}{$95 \%$ level of agreement (range) } & Minimum Significant Change \\
\cline { 2 - 9 } & Intra-raters & Inter-raters & Intra-raters & Inter-raters & Intra-raters & Inter-raters & Intra-raters & Inter-raters \\
\hline Aesthetic Index & $0.28-0.41$ & $0.17-0.28$ & $46.2-55.6$ & $37.6-41.5$ & $2 / 7(98.7-99.4 \%)$ & $2 / 7(92.5-99.4 \%)$ & $3 / 7$ & $3 / 7$ \\
\hline Shoulders & $0.42-0.53$ & $0.30-0.35$ & $64.4-73.7$ & $58.3-61.2$ & $1 / 3(99.4-100 \%)$ & $1 / 3(96.9-100 \%)$ & $2 / 3$ & $2 / 3$ \\
\hline Scapulae & $0.50-0.58$ & $0.20-0.43$ & $69.4-76.9$ & $53.5-61.9$ & $1 / 3(98.1-100 \%)$ & $1 / 3(95.6-100 \%)$ & $2 / 3$ & $2 / 3$ \\
\hline Waist & $0.48-0.70$ & $0.28-0.33$ & $75.6-82.5$ & $62.8-66.1$ & $1 / 3(100-100 \%)$ & $1 / 3(98.7-100 \%)$ & $2 / 3$ & $2 / 3$
\end{tabular}

\section{Discussion}

With this paper we aimed to develop a routine clinical tool for aesthetic evaluation of scoliosis patients, evolving from our yearly experience in grading some parameters (shoulders, scapulae, waist).

The goal was to verify the intra- and inter-rater repeatability, but most of all the sensitivity to change in a clinical setting. A secondary aim was to build on our experience to develop a new tool to be studied. Our first evaluation showed an overall fair repeatability for AI. Buchanan et al. [13] found a similar reliability for the intra- and interobserver cosmetic deformity rating among a group of orthopaedic surgeons. However, the present study revealed a low sensitivity of AI to changes; indeed, a threepoint change out of seven is the minimum change that could be considered significant. This limits the application of the AI to the detection of major changes. Therefore, we broadened all applicable parameters, determined through our experience to be easily detectable, and developed TRACE. While maintaining the same fair intra-rater repeatability, a higher sensitivity to changes is the main feature of TRACE. In fact, the 95\% level of agreement remained similar ( 2 points both for AI and TRACE for intra-rater) but the scale was now of 12 instead of 7 points (almost double that of AI): A score of three points out of twelve represents a significant change during treatment when the observer is the same. This makes TRACE much

Table 3: Correlation between Trace and Al values for the shoulder

\begin{tabular}{|c|c|c|c|c|c|c|}
\hline \multirow{2}{*}{$\mathrm{Al}$} & \multicolumn{5}{|c|}{ TRACE } & \multirow[t]{2}{*}{ Corresponding TRACE value } \\
\hline & 0 & I & 2 & 3 & TOT & \\
\hline 0 & $16 \%$ & $70 \%$ & $14 \%$ & $1 \%$ & $100 \%$ & 0.99 \\
\hline 1 & $4 \%$ & $52 \%$ & $38 \%$ & $7 \%$ & $100 \%$ & 1.48 \\
\hline 2 & $0 \%$ & $7 \%$ & $33 \%$ & $60 \%$ & $100 \%$ & 2.53 \\
\hline
\end{tabular}

more useful than AI, since it makes it possible to objectively monitor the aesthetic effects of treatment. Both AI and TRACE have been used as research tools, and we documented that TRACE is sensitive enough to detect changes induced by a brace treatment [18-20]. Moreover, this is a "no-cost" tool that can be used easily and quickly during each clinical assessment. It requires neither expensive instruments nor prolonged evaluation sessions. Usually it is sufficient to mark the sub-scale values and calculate the total TRACE score, such that in routine clinical practice photographic comparison is not needed (as we usually do since 5 years with our ISICO database software) [19,2123].

Trunk deformity significantly influences AIS patients' perception of function and self-image [24]. Therefore, both rehabilitation experts and surgeons emphasise this aspect in the decision-making process in AIS $[1,2,25]$. To date, the main outcome measures concerning the aesthetic effects of treatment are related to prominence changes [26] and the improvement of vertebral rotation [27] after brace treatment, to reduced Cobb angle after surgery $[28,29]$, or the improvement in self-perception of the deformity as assessed by questionnaire.[4] Some attempts to quantify aesthetic deformity with a clinical assessment have been performed: Theologis proposed a "Cosmetic Spinal Score (CSS)," according to which ten non-medical judges evaluated colour pictures of AIS patients [8]. The limit of this evaluation is that it gives a score pertaining to a general impression of the patient's back but is not based on precisely defined sub-scores. Nevertheless, CSS was shown to be related principally to rib hump and trunk side shift. Moreover, we have no data concerning its reliability when performed by expert physicians.

The principle that scoliosis is not simply a curvature indicated through $\mathrm{x}$-ray imaging and that there is a need for appropriate outcome measures to supplement Cobb angle has been widely recognized $[1,25]$. TRACE provides a semi-quantitative scale for clinical assessment of deformity in AIS, based on specifically defined sub-scales. 
Table 4: Correlation between Trace and Al values for waist.

\begin{tabular}{|c|c|c|c|c|c|c|c|}
\hline \multirow[t]{2}{*}{$\mathrm{Al}$} & \multicolumn{6}{|c|}{ TRACE } & \multirow[t]{2}{*}{ Corresponding TRACE value } \\
\hline & 0 & I & 2 & 3 & 4 & TOT & \\
\hline 0 & $16 \%$ & $73 \%$ & $11 \%$ & $1 \%$ & $0 \%$ & $100 \%$ & 0.96 \\
\hline 1 & $1 \%$ & $35 \%$ & $37 \%$ & $20 \%$ & $6 \%$ & $100 \%$ & 1.94 \\
\hline 2 & $1 \%$ & $1 \%$ & $15 \%$ & $44 \%$ & $39 \%$ & $100 \%$ & 3.20 \\
\hline
\end{tabular}

Table 5: Correlation between Trace and Al total values

\begin{tabular}{|c|c|c|c|c|c|c|c|c|c|c|c|c|c|c|}
\hline \multirow[t]{2}{*}{$\mathrm{Al}$} & \multicolumn{13}{|c|}{ TRACE } & \multirow[t]{2}{*}{ Corresponding TRACE value } \\
\hline & 1 & 2 & 3 & 4 & 5 & 6 & 7 & 8 & 9 & 10 & 11 & 12 & TOT & \\
\hline 0 & $0 \%$ & $17 \%$ & $38 \%$ & $42 \%$ & $4 \%$ & $0 \%$ & $0 \%$ & $0 \%$ & $0 \%$ & $0 \%$ & $0 \%$ & $0 \%$ & $100 \%$ & 2.33 \\
\hline 1 & $0 \%$ & $2 \%$ & $15 \%$ & $30 \%$ & $28 \%$ & $12 \%$ & $9 \%$ & $2 \%$ & $1 \%$ & $0 \%$ & $0 \%$ & $0 \%$ & $100 \%$ & 3.73 \\
\hline 2 & $0 \%$ & $0 \%$ & $4 \%$ & $19 \%$ & $27 \%$ & $26 \%$ & $14 \%$ & $6 \%$ & $1 \%$ & $1 \%$ & $0 \%$ & $0 \%$ & $100 \%$ & 4.56 \\
\hline 3 & $0 \%$ & $0 \%$ & $2 \%$ & $6 \%$ & $20 \%$ & $26 \%$ & $24 \%$ & $12 \%$ & $7 \%$ & $3 \%$ & $1 \%$ & $0 \%$ & $100 \%$ & 5.44 \\
\hline 4 & $0 \%$ & $0 \%$ & $0 \%$ & $1 \%$ & $6 \%$ & $15 \%$ & $29 \%$ & $25 \%$ & $14 \%$ & $4 \%$ & $4 \%$ & $1 \%$ & $100 \%$ & 6.56 \\
\hline 5 & $0 \%$ & $0 \%$ & $0 \%$ & $0 \%$ & $0 \%$ & $2 \%$ & $14 \%$ & $9 \%$ & $22 \%$ & $22 \%$ & $30 \%$ & $2 \%$ & $100 \%$ & 8.44 \\
\hline 6 & $0 \%$ & $0 \%$ & $0 \%$ & $0 \%$ & $0 \%$ & $0 \%$ & $14 \%$ & $0 \%$ & $0 \%$ & $7 \%$ & $64 \%$ & $14 \%$ & $100 \%$ & 9.50 \\
\hline
\end{tabular}

Knowing the limits and the repeatability of this scale will give clinicians more reliability in the routine clinical assessment of deformity, and will provide other sensitive outcome measures. TRACE is consistent with this need, and it can readily be used in the clinical evaluation of AIS patients and for research.

One limitation of this study was the use of pictures instead of an immediate evaluation of patients. Nevertheless, evaluation through pictures has been the standard applied in previous studies, $[2,4,5]$ and we can presume the repeatability to be even greater during routine clinical practice due to the opportunity for a three-dimensional evaluation of the patient. In fact, photographs are static while a three-dimensional clinical assessment presumably can be more consistently recorded by the physician, and in future studies can provide a tool to compare TRACE with the POTSI index. Another limit could be the low Kappa Statistics values obtained, even if comparable to those obtained by others evaluating aesthetics previously[8]; but is less important for clinical routine use than the minimum significant change.
This study documents the evolution of TRACE from AI. TRACE is sufficiently repeatable and sensible for routine clinical practice, and therefore comprises is a no-cost tool designed for the conservative clinical setting.

\section{Competing interests}

The authors declare that they have no competing interests.

\section{Authors' contributions}

All authors made substantial contributions to conception, design and acquisition of data; they have been involved in drafting and revising the manuscript; they have given final approval of the version to be published.

\section{Acknowledgements}

The Authors wish to thank Dr. Chiara Paroli and Dr. Marco Monticone for their kind help and collaboration in collecting data for this study.

The Authors wish to thank the "Officina Ortopedica Ortho Tecnica" - Via IV Novembre 93/B, 38014 GARDOLO (TN) of Fabrizio Tessadri for his contribution to covering the cost of this publication. 


\section{References}

I. Negrini S, Grivas TB, Kotwicki T, Maruyama T, Rigo M, Weiss HR: Why do we treat adolescent idiopathic scoliosis? What we want to obtain and to avoid for our patients. SOSORT 2005 Consensus paper. Scoliosis 2006, I:4.

2. Donaldson S, Hedden D, Stephens D, Alman B, Howard A, Narayanan $U$, Wright JG: Surgeon reliability in rating physical deformity in adolescent idiopathic scoliosis. Spine 2007, 32(3):363-367.

3. Asher M, Min Lai S, Burton D, Manna B: The reliability and concurrent validity of the scoliosis research society-22 patient questionnaire for idiopathic scoliosis. Spine 2003, 28(I):63-69.

4. Sanders JO, Polly DW Jr, Cats-Baril W, Jones J, Lenke LG, O'Brien MF, Stephens Richards B, Sucato DJ: Analysis of patient and parent assessment of deformity in idiopathic scoliosis using the Walter Reed Visual Assessment Scale. Spine 2003, 28(I 8):2| 58-2|63.

5. Sanders JO, Harrast JJ, Kuklo TR, Polly DW, Bridwell KH, Diab M, Dormans JP, Drummond DS, Emans JB, Johnston CE 2nd, et al.: The Spinal Appearance Questionnaire: results of reliability, validity, and responsiveness testing in patients with idiopathic scoliosis. Spine 2007, 32(24):2719-2722.

6. Weisz I, Jefferson RJ, Turner-Smith AR, Houghton GR, Harris JD: ISIS scanning: a useful assessment technique in the management of scoliosis. Spine 1988, I 3(4):405-408.

7. Weisz I, Jefferson RJ, Carr AJ, Turner-Smith AR, Mclnerney A Houghton GR: Back shape in brace treatment of idiopathic scoliosis. Clin Orthop Relat Res 1989:157-163.

8. Theologis TN, Jefferson RJ, Simpson AH, Turner-Smith AR, Fairbank JC: Quantifying the cosmetic defect of adolescent idiopathic scoliosis. Spine 1993, I 8(7):909-9|2.

9. Liu XC, Thometz JG, Lyon RM, Klein J: Functional classification of patients with idiopathic scoliosis assessed by the Quantec system: a discriminant functional analysis to determine patient curve magnitude. Spine 200 I, 26(I I): | 274- I 278. discussion 1279 .

10. Hackenberg L, Hierholzer E, Potzl W, Gotze C, Liljenqvist U: Rasterstereographic back shape analysis in idiopathic scoliosis after anterior correction and fusion. Clin Biomech (Bristol, Avon) 2003, I 8(I): I-8.

II. Negrini A, Negrini S, Santambrogio GC: Data variability in the analysis of spinal deformity, a study performed by means of the AUSCAN system. In Three Dimensional Analysis of Spinal Deformities Edited by: D'Amico M, Merolli A, Santambrogio GC. Amsterdam: IOS Press; 1995: I0I-106.

12. Negrini S, Negrini A, Rainero G, Sibilla P, Santambrogio GC: Correlation Between Trunk Gibbosity and the Spinal Torsion Measured by the AUSCAN System. In Three Dimensional Analysis of Spinal Deformities Volume I. Edited by: D'Amico M, Merolli A, Santambrogio GC. Amsterdam: IOS Press - Ohmsha; 1995:279-283.

13. Buchanan R, Birch JG, Morton AA, Browne RH: Do you see what I see? Looking at scoliosis surgical outcomes through orthopedists' eyes. Spine 2003, 28(24):2700-2704. discussion 2705.

14. Cohen J: A coefficient of agreement for nominal scales. Educational and Psychological Measurement 1960, 20:37-46.

I5. Fleiss J: Measuring nominal scale agreement among many raters. Psychological Bulletin 1971, 76:378-38I.

16. Maclure M, Willett $W$ : Misinterpretation and misuse of the kappa statistic. American Journal of Epidemiology 1987, I 26: I6I-169.

17. Uebersax JS: Modeling approaches for the analysis of observer agreement. Invest Radiol 1992, 27(9):738-743.

18. Zaina F, Negrini S, Atanasio S: Brace treatment can change aesthetics in Adolescent Idiopathic Scoliosis (AIS) patients. In 5th International Conference on Conservative Management of Spinal Deformities: 2008 Athens: SOSORT; 2008

19. Negrini S, Atanasio S, Zaina F, Romano M, Parzini S, Negrini A: Endgrowth results of bracing and exercises for adolescent idiopathic scoliosis. Prospective worst-case analysis. Stud Health Technol Inform 2008, I 35:395-408.

20. Zaina F, Negrini S, Monticone M, Paroli C: TRACE (Trunk Aesthetic Clinical Evaluation), a new everyday clinical tool to assess adolescent idiopathic scoliosis patients aesthetics. In 4th International Conference on Conservative Management of Spinal Deformities: 13-16 May 2007 Boston: SOSORT (Society on Scoliosis Orthopaedic and Rehabilitation Treatment); 2007.

21. Negrini S, Atanasio S, Fzaina F, Romano M: Rehabilitation of adolescent idiopathic scoliosis: results of exercises and bracing from a series of clinical studies. Europa Medicophysica-SIMFER 2007 Award Winner. Eur J Phys Rehabil Med 2008, 44(2): $169-176$.

22. Negrini S, Atanasio S, Negrini F, Zaina F, Marchini G: The Sforzesco brace can replace cast in the correction of adolescent idiopathic scoliosis: A controlled prospective cohort study. Scoliosis 2008, 3(I): I5.

23. Negrini S, Zaina F, Romano M, Negrini A, Parzini S: Specific exercises reduce brace prescription in adolescent idiopathic scoliosis: A prospective controlled cohort study with worst-case analysis. J Rehabil Med 2008, 40(6):45 I-455.

24. Asher M, Lai SM, Burton D, Manna B: The influence of spine and trunk deformity on preoperative idiopathic scoliosis patients' health-related quality of life questionnaire responses. Spine 2004, 29(8):86I-868.

25. Turner-Smith AR, Harris JD, Houghton GR, Jefferson RJ: A method for analysis of back shape in scoliosis. J Biomech 1988 , 2 I (6):497-509.

26. Grivas TB, Vasiliadis ES: Cosmetic outcome after conservative treatment of idiopathic scoliosis with a dynamic derotation brace. Stud Health Technol Inform 2008, I35:387-392.

27. Kotwicki T, Kinel E, Stryla W, Szulc A: Discrepancy in clinical versus radiological parameters describing deformity due to brace treatment for moderate idiopathic scoliosis. Scoliosis 2007, 2: 18

28. Cochran T, Irstam L, Nachemson A: Long-term anatomic and functional changes in patients with adolescent idiopathic scoliosis treated by Harrington rod fusion. Spine I983, 8(6):576-584.

29. Weinstein SL, Dolan LA, Cheng JC, Danielsson A, Morcuende JA: Adolescent idiopathic scoliosis. Lancet 2008, 37 I(9623): I 527-I 537.
Publish with Biomed Central and every scientist can read your work free of charge

"BioMed Central will be the most significant development for disseminating the results of biomedical research in our lifetime. "

Sir Paul Nurse, Cancer Research UK

Your research papers will be:

- available free of charge to the entire biomedical community

- peer reviewed and published immediately upon acceptance

- cited in PubMed and archived on PubMed Central

- yours - you keep the copyright
BioMedcentral 